\title{
Research on preparation of chrome tin purple red pigment by using eggshell
}

The solid-phase synthesis of Cr-Ca-Sn-Si purple red pigment, the preparation of chrome tin pink stains are studied by using eggshell, which is used as the raw materials introducing the calcium carbonate. Through the experiment of formula is optimized, and by controlling the calcinations temperature and mineralizer measures a series of $\mathrm{Cr}-\mathrm{Ca}-\mathrm{Sn}$ Si purple red pigments are prepared. The results showed that $\mathrm{Cr}-\mathrm{Ca}-\mathrm{Sn}$-Si purple red pigments are prepared by using eggshell.

Keywords: Eggshell; Cr-Ca-Sn-Si purple red pigments; solid-phase method.

\subsection{Introduction}

$\mathrm{C}$ hrome tin purple red ( $\mathrm{Cr}-\mathrm{Ca}-\mathrm{Sn}-\mathrm{Si}$ ) ceramic pigment is a kind of rare red materials which are favored by most people because of its beautiful color. Since the 1980s, especially after the popular usage of gas and liquid fuel, company with the introduction of oxidation atmosphere which can be strictly controlled, red chrome-tin ceramic pigment has been widely used for the products of high-grade sanitary ware and tiles. As a result, material research is quite active in recent years. There is no doubt that red chrome-tin has high stability of crystal type and is widely used in highgrade sanitary ceramics and ceramic pigment red series [1].

Chrome-tin red (Cr-Ca-Sn-Si) pigment is a kind of red material with purple hues, also known as red agate, red garden and so on. In terms of its load color matrix, it can be divided into perovskite type and sphene type, which are named as the ash tin $\left(\mathrm{CaSnO}_{3}\right)$ and sphene $\left(\mathrm{CaSnSiO}_{5}\right)$ correspondingly. The color of chrome-tin red (Cr-Ca-Sn-Si) pigment is colorful, which can be shown as deep red, rosy, purple, lilac and so on. It is more than a half century since this kind of red material was discovered.

Calcium carbonate is one of the main raw materials of chrome tin purple red, and the shell contains a lot of $\mathrm{CaCO}_{3}$, which is very cheap raw materials. It is shown that our country egg production has been held for 20 years all over

Blind peer reviews carried out

Messrs. Hongyan Zhang and Jinhua Wang, Qinggong College, North China University of Science and Technology, Tangshan 063000, China the world [2]. On the one hand, using eggshell to make ceramic pigment can purify environment, on the other hand, synthetic pigment with high added value can produce remarkable social benefits and economic benefits. Chrome-tin red material made by Zhao Yan zhao can be comparable to those $\mathrm{f}$ commercialized pigment, which not only effectively reduced the cost, but also increased the economic returns [3]. This article introduced eggshell calcium carbonate raw material, using the method of solid phase synthesis of $\mathrm{Cr}-\mathrm{Ca}$ $\mathrm{Sn}-\mathrm{Si}$ amaranth, and a series of the purple materials are also produced by adjusting the formula, calcinations system and mineralize measures.

\subsection{The research status at home and abroad}

For decades, scholars at home and abroad have conducted many experiments about the preparation process of chrome tin amaranth and glaze, color mechanism, basic glaze type, the type of additives, and firing atmosphere for a wide range of research. The concrete content is as follows: as early as 1936 , Mellor considered chrome oxide micelle fine dispersed in tin oxide and color, pigment color depends on the ratio of the chromic oxide and tin oxide, from deep red to rose red, and the effect of calcium oxide was to make the pink color is better, and more stable in high temperature. But due to the instability of chrome oxide particles in the heat air, the theory is not convincible. In the 50s, the Japanese scholar Jin Gang garten put forward a theory about the $\mathrm{Cr}-\mathrm{Sn}$ series pigment with solid solution. In 1968, the Soviet scholars put forward a theory about free $\mathrm{Cr}_{3}+$ solution color in the tin sphene crystal structure. At that time, the two theories have not been confirmed by experiment and unconvincing. Until 1986 Japanese scholar Shi Tian Xinwu, using modern testing methods, firstly proposed the chemical valence of chromium ion in tin scrap stone instead of $\mathrm{Cr}_{3}+, \mathrm{Cr}_{4}+$. In 1994, Richard. Eppler pointed out in his paper that the chrome-tin red pigment produced by $\mathrm{Cr}_{2} \mathrm{O}_{3}$ and $\mathrm{SnO}_{2}$ system will appear the color of orchid. After adding $\mathrm{CaO}$ and $\mathrm{SiO}_{2}$, sphene crystal structure type can produce pink, red, pink, Venetian red, purple, green and other colors, among which the red is the most close to the red. Chinese scholars Yu Kang tai and Xu Xiao hong have gotten the same conclusion obtained by experiment recently. They have pointed out that chrome-tin 
showing red is due to chromium solid fusion in $\mathrm{CaO} \cdot \mathrm{SnO}_{2} \cdot \mathrm{SiO}_{2}$ sphene crystal structure; chromium ion in the chemical valence state is $\mathrm{Cr}_{4}+$ tin titanite, rather than $\mathrm{Cr}_{3}+$; and they further proposed that agate red material is the main mineral composition of tin sphene. And in pigment, the more tin sphene is, the better is the color. Agate red glaze must be burnt in the oxidizing atmosphere, the main chemical valence of $\mathrm{Cr}_{4}+$ chrome. Its color is due to the doped chromium, and in the process, $\mathrm{Cr}_{4}+$ substitution tin sphene crystal lattice $\mathrm{Sn}_{4}+$, making $\left(\mathrm{SnO}_{6}\right)$ octahedral reformat and the lattice distort, which can lead to the cause pigment in the reflection spectrum of visible light around $520 \mathrm{~nm}$ formed a wide absorption band, so that the agate red glaze appears.

At present, main synthetic methods of $\mathrm{Cr}-\mathrm{Ca}-\mathrm{Sn}-\mathrm{Si}$ gezer are solid instead should method, chemical coprecipitation method, sol-gel method, etc. The advantages of solid instead method are its simple process, short production cycle and low cost. The disadvantage is that reaction rate is affected by diffusion and the stability of the pigment is difficult to control. Chemical co precipitation is strict about process control, and it is easy to produce precipitation sequence leading to component segregation and ultimately affect the accuracy of the results. The advantages of Sol-gel method are that uniform dispersion agent and color effect is good. While the disadvantages are the high cost, unstable and immature technology. The color of Cr-Ca-Sn-Si gezer would change from lavender, pink, agate red, red, purple to red with the different ratio of raw materials, which must be burnt in the oxidizing atmosphere.

Nearly 20 years, the research achievements of domestic chrome-tin red material basically are: Yu Kang tai and Zuo Quan carried a study of producing chrome-tin red material by sol gel method in 1998. In the same year Yu Kang tai, Hong Xiao lin and Gao Sheng zhou carried a study of producing chrome-tin red material in the chemical coprecipitation preparation chrome-tin red materials research. In 2001, Zheng Nai zhang, Xiong Chun hua and Zhu Zhi gang has carried out a study on the synthetic technology of $\mathrm{Cr}-\mathrm{Ca}-\mathrm{Sn}-\mathrm{Si}$ series of purple red color. Zhou Li juan conducted the research about the low temperature synthesis of chrome-tin red material and its optical properties in 2002. Wang Jin, Song Shu'e adopted alcohol - water solution coprecipitation preparation to make chrome-tin red ceramic pigment superfine powder in 2004. Lang Xian kun, Lin Wei and Li Xiao sheng carried out a study on producing chrome-tin red material by solid method in 2015.

One of the main raw materials of chrome tin purple material is calcium carbonate, which can be contained a lot of $\mathrm{CaCO}_{3}$ in the eggshell. China is the most populous nation in the world with the greatest amount of hens. The egg production has been held for 20 years in the world. According to statistics, China's egg production is 25.607 million in 2003, per capita is $19.5 \mathrm{~kg}$. Egg consumption per capita are more than the world average. Each egg production accounted for more than $43 \%$ of the world's total egg production. Having abundant eggshell resources in the country, the factory in which the manufacturer is processing using eggshell is seldom. Eggshell research development is also very weak. The economic benefits will be dozens of times, even hundreds if the eggshell various ingredients are used to carry on the comprehensive utilization of modern industrial production and be developed as forage products, edible, medicinal and cosmetic products. On one hand, using eggshell preparation of ceramic pigment can purify environment, on the other hand, synthetic pigment with high added value can produce remarkable social benefits and economic benefits. Zhao Yan zhao used shell to make chrome-tin red material which can be comparable to those of commercialization, not only effectively reducing the cost, but increasing economic benefits.

\subsection{Experiment}

\subsection{Materials AND INSTRUMENTS}

Tin oxide $\left(\mathrm{SnO}_{2}\right)$, silicon dioxide $\left(\mathrm{SiO}_{2}\right)$, potassium dichromate $\left(\mathrm{K}_{2} \mathrm{Cr}_{2} \mathrm{O}_{7}\right)$, borax $\left(\mathrm{Na}_{2} \mathrm{~B}_{4} \mathrm{O}_{7} \cdot 10 \mathrm{H}_{2} \mathrm{O}\right)$, fluorite $\left(\mathrm{CaF}_{2}\right)$, anhydrous ethanol $\left(\mathrm{C}_{2} \mathrm{H}_{5} \mathrm{OH}\right)$, concentrated hydrochloric acid $(\mathrm{HCl})$, eggshell (collected in daily life)

Electric drum wind drying oven, high temperature box type resistance furnace, electronic scale, analytical balance

\subsection{EXPERIMENT PROCESS}

\subsubsection{The composition of the shell}

The main chemical compositions are listed in Table 1[4]. Table 1 shows $\mathrm{CaCO}_{3}$ in eggshell was more than $93 \%$, the $\mathrm{Ca}$ element content is greater than $36 \%$, far higher than that of animal bone calcium content (12\%). Eggshell organic matter content is very low, so it is suitable for the preparation of chromium tin purple material.

Table 1: Chemical Composition of EgGShell Content (wT\%)

\begin{tabular}{lcccc}
\hline Component & $\mathrm{CaCO}_{3}$ & $\mathrm{MgCO}_{3}$ & $\mathrm{Ca}\left(\mathrm{PO}_{4}\right)_{2}$ & Organic compound \\
\hline Content & 93.0 & 1.0 & 2.8 & 3.2 \\
\hline
\end{tabular}

\subsubsection{The pretreatment of the eggshell}

After watering and drying the collected shells with distilled water, put them into the oven to dry and then crush them. The combination of shell and shell membrane is essentially a combination of calcareous and keratin. In order to separate shell and shell membrane better, hydrochloric acid (12 mol/L) that can reduce the bonding force between calcareous and keratin concentrated and distilled water can be used.

\subsubsection{The preparation process of the pigment}

The process is shown in Fig. 1, with $\mathrm{SnO}_{2}, \mathrm{SiO}_{2}, \mathrm{~K}_{2} \mathrm{Cr}_{2} \mathrm{O}_{7}$, shell as raw materials, with $\mathrm{K}_{2} \mathrm{Cr}_{2} \mathrm{O}_{7}$ as coloring agent, borax $\left(\mathrm{Na}_{2} \mathrm{~B}_{4} \mathrm{O}_{7} \cdot 10 \mathrm{H}_{2} \mathrm{O}\right)$ and fluorite mineralization agent $\left(\mathrm{CaF}_{2}\right)$, by adjusting the proportion of different mixture, mix with agate 


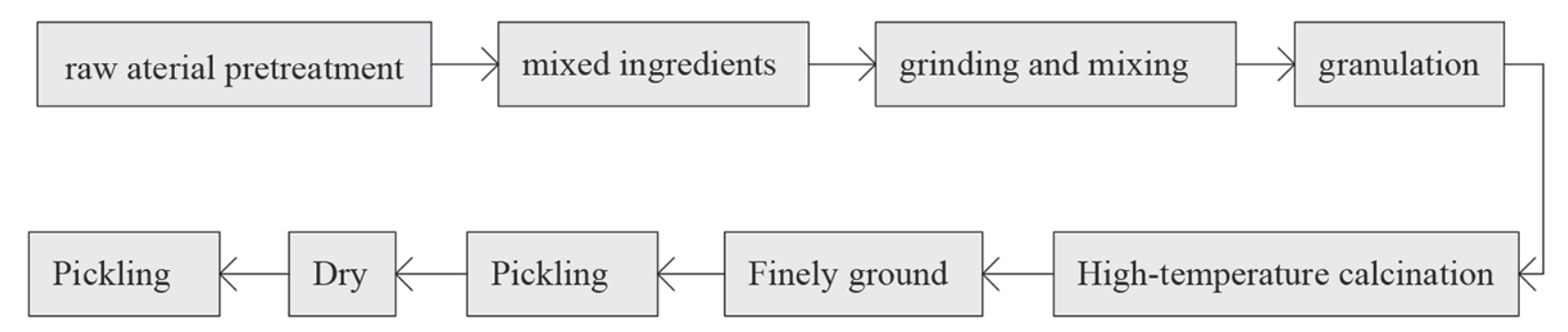

Fig.1: Process flow chart

TABLE 2: $\mathrm{SNO}_{2}$ EXCESS FORMULA DESIGN (WT\%)

\begin{tabular}{ccccccc}
\hline Number & $\mathrm{SnO}_{2}(\%)$ & Eggshell (\%) & $\mathrm{SiO}_{2}(\%)$ & $\mathrm{K}_{2} \mathrm{Cr}_{2} \mathrm{O}_{7}(\%)$ & $\mathrm{Na}_{2} \mathrm{~B}_{4} \mathrm{O}_{7} \cdot 10 \mathrm{H}_{2} \mathrm{O}(\%)$ & $\mathrm{Coloration}$ \\
\hline 1 & 50.6 & 32.0 & 19.4 & 5.6 & 4.0 & dark purple \\
2 & 50.2 & 32.0 & 19.4 & 5.6 & 4.0 & deep purple \\
3 & 49.8 & 32.0 & 19.4 & 5.6 & 4.0 & purple \\
4 & 49.4 & 32.0 & 19.4 & 5.6 & 4.0 & purple \\
5 & 49.0 & 32.0 & 19.4 & 5.6 & 4.0 & purple red \\
6 & 48.6 & 32.0 & 19.4 & 5.6 & purple red \\
\hline
\end{tabular}

mortar grinding, through 250 mesh sieve, claimed at certain temperatures and heat preservation 2 hours after natural cooling, then observe the sample.

The preparation of graphene oxide from natural graphite powder was following the modified Hummers-Offeman method. In short, the graphite powder $(9.8 \mathrm{~g})$ was dispersed in concentrated sulfuric acid $(230 \mathrm{~mL}, 98 \%)$ cooled in an ice bath. Then the potassium permanganate $\left(\mathrm{KMnO}_{4}, 30 \mathrm{~g}\right)$ was gradually added with stirring. We should pay attention to avoid the temperature of the mixture from exceeding $293 \mathrm{~K}$ by cooling. After that, the ice bath was removed and replace with a water bath and the mixture was heated to $307 \mathrm{~K}$ under the continuous stirring in $40 \mathrm{~min}$. After $40 \mathrm{~min}$ reaction, we slowly added the deionized water $(480 \mathrm{~mL})$ caused a rapid increase in solution temperature up to the maximum of $372 \mathrm{~K}$. In order to make the graphite powder fully react, the reaction was maintained at $372 \mathrm{~K}$ for $40 \mathrm{~min}$. A bright yellow suspension was obtained by the addition of distilled water $(240 \mathrm{~mL})$ and a solution of hydrogen peroxide $(30 \%, 250 \mathrm{~mL})$ respectively. The precipitate was separated by centrifugation at $3000 \mathrm{r} / \mathrm{min}$, and then washed with hydrochloric acid several times. The solid was washed five times with acetone and dried $24 \mathrm{~h}$ in a vacuum oven.

\subsection{The results and discussions of experimentene}

\subsection{OptimizaTION OF FORMULA}

The composition of raw materials has a great influence on the $\mathrm{Cr}-\mathrm{Ca}-\mathrm{Sn}-\mathrm{Si}$ of the chromium (tin)/purple color. This experiment according to tin sphene formula $\mathrm{CaO} \cdot \mathrm{SnO}_{2} \cdot \mathrm{SiO}_{2}$, takes mole ratio of $\mathrm{CaO}: \mathrm{SnO}_{2}: \mathrm{SiO}_{2}=1: 1: 1$, calculation of eggshell, silicon dioxide, the quality of the tin oxide, its ingredients $\mathrm{SnO}_{2} 48.6 \%$, eggshell $32.0 \%, \mathrm{SiO}_{2} 19.4 \%$. In the experiment, a series of amaranth can be produced by changing the formula of tin oxide and shell of content, content of formula of borax is $4 \%$, potassium dichromate adding amount is $5.6 \%$.

\subsubsection{The influence of $\mathrm{SnO}_{2}$ dosage on color}

Experiments adopt potassium dichromate as coloring agent, borax as the mineralizer. And the blending ratio and the experimental results are shown in Table 2, according to the Fig.1, all the materials are in the $1250^{\circ} \mathrm{C}$ calcinations, insulated natural cooling to room temperature after 2 hours, observe the color, the experiment result is shown in Fig.2.

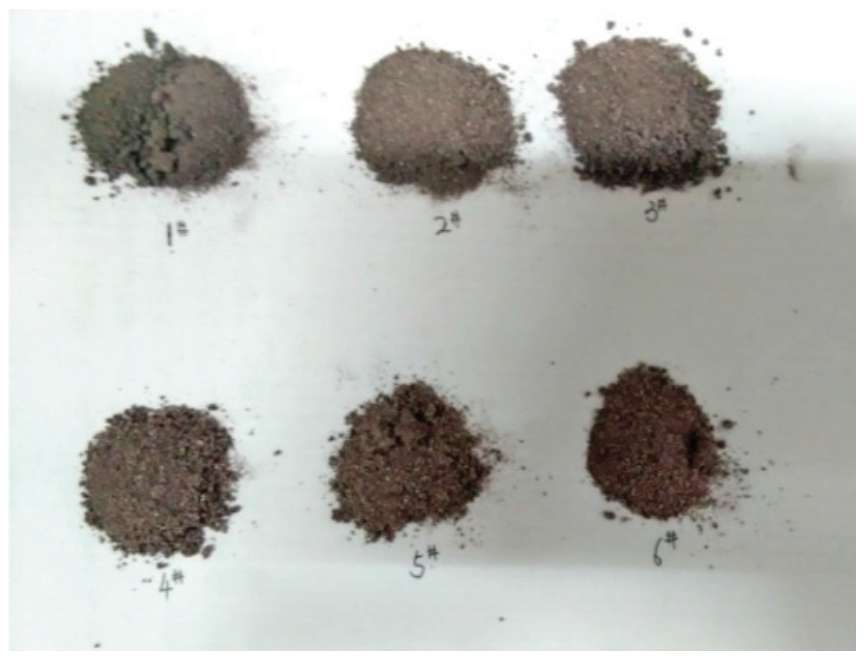

Fig.2: The preparation of pigments with different $\mathrm{SnO}_{2}$ contents

In Fig.2, the 1\#-6\# are corresponding to formula 1-6 in Table 2. As can be seen from the Fig.2, when excessive content of tin oxide can be produced in color dark purple, purple, violet, and purple, and a series of purple colored pigments. 
TABLE 3: EgGSHELl EXCESS FORMULA DESIGN (WT\%)

\begin{tabular}{ccccccc}
\hline Number & $\mathrm{SnO}_{2}(\%)$ & Eggshell $(\%)$ & $\mathrm{SiO}_{2}(\%)$ & $\mathrm{K}_{2} \mathrm{Cr}_{2} \mathrm{O}_{7}(\%)$ & $\mathrm{Na}_{2} \mathrm{~B}_{4} \mathrm{O}_{7} \cdot 10 \mathrm{H}_{2} \mathrm{O}(\%)$ & $\mathrm{Coloration}$ \\
\hline 1 & 48.6 & 34.5 & 19.4 & 5.6 & 4.0 & 4.0 \\
2 & 48.6 & 34.0 & 19.4 & 5.6 & 4.0 & dark purple \\
3 & 48.6 & 33.5 & 19.4 & 5.6 & 5.0 & purple \\
4 & 48.6 & 33.0 & 19.4 & 5.6 & 4.0 & purple \\
5 & 48.6 & 32.5 & 19.4 & 5.6 & 4.0 & purple red \\
6 & 48.6 & 32.0 & 19.4 & 5.6 & purple red \\
\hline
\end{tabular}

Table 4: Percentage content of Potassium dichromate (wT\%)

\begin{tabular}{|c|c|c|c|c|c|c|}
\hline Number & $\mathrm{SnO}_{2}(\%)$ & Eggshell (\%) & $\mathrm{SiO}_{2}(\%)$ & $\mathrm{K}_{2} \mathrm{Cr}_{2} \mathrm{O}_{7}(\%)$ & $\mathrm{Na}_{2} \mathrm{~B}_{4} \mathrm{O}_{7} \cdot 10 \mathrm{H}_{2} \mathrm{O}(\%)$ & Coloration \\
\hline 1 & 48.6 & 32.0 & 19.4 & 4.6 & 4.0 & deep purple \\
\hline 2 & 48.6 & 32.0 & 19.4 & 1.6 & 4.0 & flesh pink \\
\hline 3 & 48.6 & 32.0 & 19.4 & 0 & 4.0 & milky \\
\hline
\end{tabular}

\subsubsection{Eggshell dosage effect on color}

In the experiment, potassium dichromate is used as coloring agent, borax as the mineralizer. And blending ratio and the experimental results are shown in Table 3 . According to the Fig.1, the sample is calcinated in the $1250^{\circ} \mathrm{C}$, insulated natural cooling to room temperature after 2 hours, observe the color, the experiment results are shown in Fig.3.

Fig.3, the sample number 1 to number 6 are corresponding to the formula one to six in Table 3 . As can be seen from the Fig.3, the content of the eggshells can produce dark purple, purple, violet, and purple, and a series of purple colored pigments.

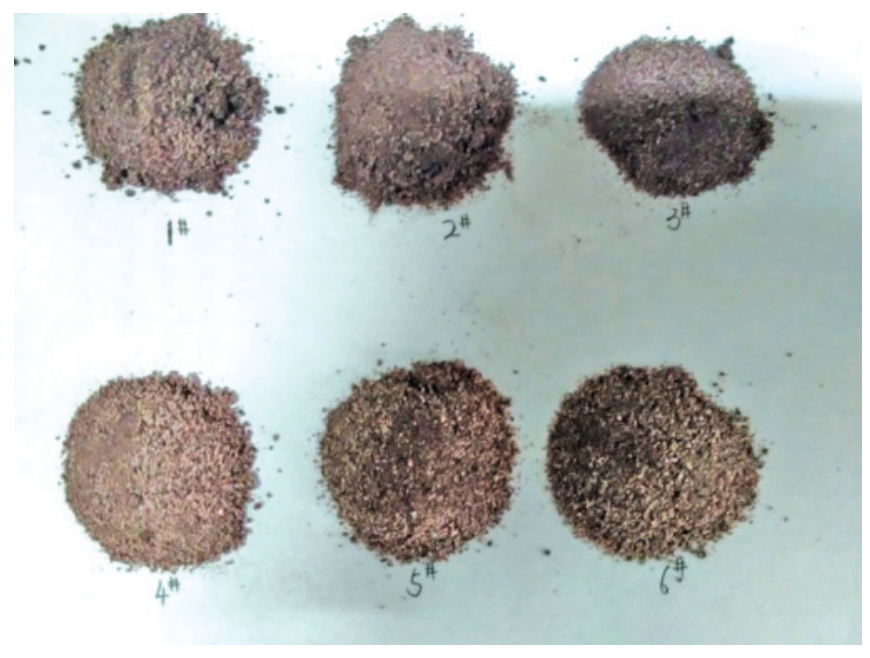

Fig.3: The content of pigments in different eggshells

\subsubsection{Colorant dosage of potassium dichromate effect on color}

Experiments use potassium dichromate as coloring agent, borax as the mineralizer. The blending ratio and the experimental results are shown in Table 4. According to the Fig. 1 process preparation, the sample is calcinated in the $1250^{\circ} \mathrm{C}$, insulation natural cooling to room temperature after 2

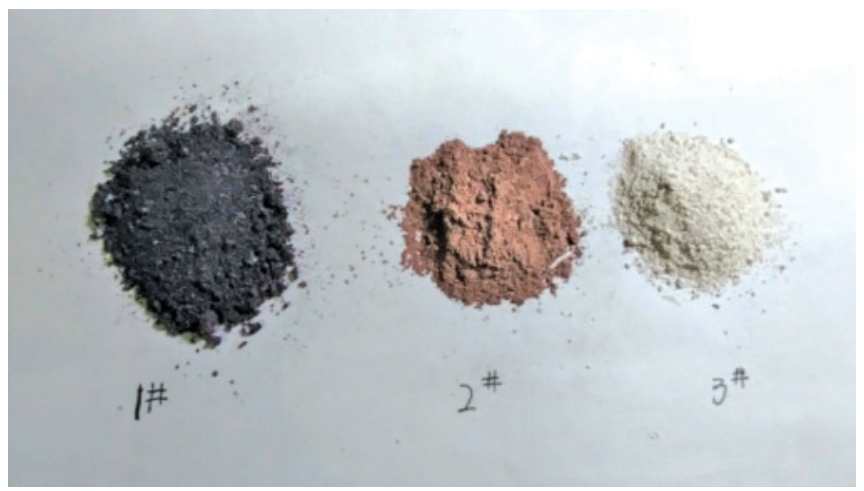

Fig.4: The pigments prepared by different contents of potassium dichromate

hours, then observe the color, the experiment result is shown in Fig.4.

Fig.4, the 1\#-3\# are corresponding with formula 1-3 in Table 4. In the formula, the content of chromium has a great influence on the color pigment. The experimental results show that when the chromium content at $1.6 \% \sim 5.6 \%$, with purple colored pigments can be prepared, when it does not contain chromium pigment, the pigment appears as white.

\subsection{THE CHOICE OF MINERALIZER}

In ceramic color agent synthetic reaction, common compound mineralizers are boron compounds, fluorine, chlorine and alkali oxides. The reason of mineralizer speeding up the solid instead lies in that mineralizer, in some cases, can melt into liquid phase, contribute to the diffusion and reaction speed; and it also can reduce the viscosity of the liquid phase, speed up the reaction diffusion speed and make the response speed [5]. The experiment adopts the mineralizer as main two kinds of $\mathrm{Na}_{2} \mathrm{~B}_{4} \mathrm{O}_{7} \cdot 10 \mathrm{H}_{2} \mathrm{O}$ and $\mathrm{CaF}_{2}$, by adding different mineralizer to formulation, to prepare chromium calcine tin amaranth, according to the product of the shading conditions to choose the appropriate colorants.

Mineralizer has a great influence on pigment color. When 
using mineralizer as calcium fluoride, the pigment particles are smaller and a degree of particle size distribution is more homogeneous. When using $\mathrm{Na}_{2} \mathrm{~B}_{4} \mathrm{O}_{7} \cdot 10 \mathrm{H}_{2} \mathrm{O}$ as mineralizer, the pigment particles is larger, a degree of particle size distribution is wider. In Fig.5, the $1 \#$ mineralizer is calcium fluoride, $2 \#$ mineralizer is $\mathrm{Na}_{2} \mathrm{~B}_{4} \mathrm{O}_{7} \cdot 10 \mathrm{H}_{2} \mathrm{O}$.

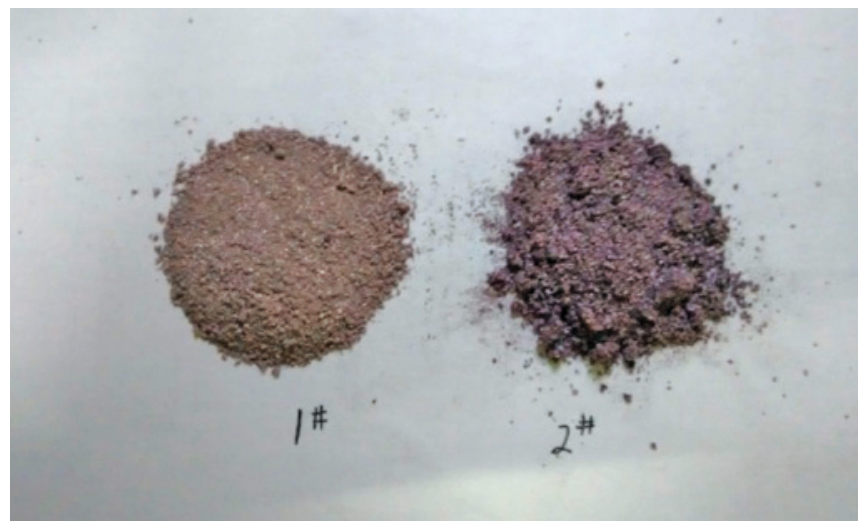

Fig.5: Pigment prepared by calcining different mineralizer

\subsection{THE DETERMINATION OF PIGMENT CALCINATION TEMPERATURE}

The influence of temperature is the same as the usual chemical reaction, raising the temperature can improve the speed of the solid phase reaction. This experiment is on the basis of the same formula ingredients and transfer to porcelain crucible after the grinding then put in resistance furnace heating. The temperature is set at $1200^{\circ} \mathrm{C}$ and $1250^{\circ} \mathrm{C}$ respectively, the experimental results are shown in Fig.6.

The pigment color prepared by different calcination

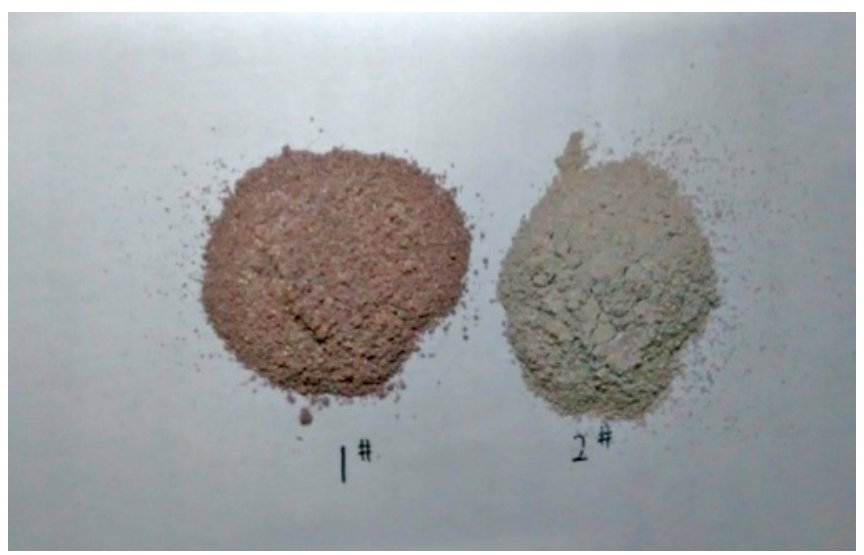

Fig.6: Pigment prepared at different calcination temperature temperature has the very big difference. When the formula is the same, the higher the temperature is, the deeper will be the pigment color. If the calcination temperature is reduced, the ink color will become lighter. In Fig.6, 1\# calcination temperature is $1250^{\circ} \mathrm{C}, 2 \#$ calcination temperature is $1200^{\circ} \mathrm{C}$. Calcination preparation of pigment color is deeper purple when the temperature is $1250^{\circ} \mathrm{C}$.

\subsection{Conclusions}

1. A series of $\mathrm{Cr}-\mathrm{Ca}-\mathrm{Sn}-\mathrm{Si}$ (chromium tin) purple red pigments, can also be prepared using eggshell as raw material instead of calcium carbonate, with the advantage of savings cost and purifying the environment.

2. Cr-Ca-Sn-Si (chrome tin purple) color of the purple is associated with the content of tin oxide and calcium oxide, and the content of potassium dichromate colorant exert influences.

3. When using solid phase sintering to produce the $\mathrm{Cr}-\mathrm{Ca}$ $\mathrm{Sn}-\mathrm{Si}$ (chromium tin) purple red pigments, selection of mineralizer and calcination temperature both affect the coloring material color.

\section{Acknowledgments}

This work is supported by Hebei Province Science and Technology Plan Projects (No.15211119).

\section{References}

1. Lang Xian kun, Lin Wei, Li Xiao sheng, etc. (2015): Research of chrome-tin red pigment preparation using solid phase method [J]. Journal of Qiqihar University, 3:27-29.

2. Bin Dong mei, Ma Mei hu, Yi Cheng. (2006): Eggshell comprehensive utilization of resources and the status quo Approach[J]. Food Research and Development, 8:212-216.

3. Zhao Yan Zhao, Song Hong li, Wu Xiao dong, etc. (2004): Research on preparation of chrome tin pink stains by using eggshell[J]. Journal of Shaanxi University of Science and Technology, (5):113-115.

4. Zhang Rui yu. (2006): Utilizing value of wasted eggshell and its resource-making way and technique[J]. Chongqing Technology Business University (Natural Science), 12:551.

5. Jang HM, ChoSR. Lee K M. (1995): Machanism of formation $\mathrm{Pb}\left((\mathrm{Zn}, \mathrm{Mg}) 1 / 3 \mathrm{Nb}_{2} / 3\right) \mathrm{O}_{3}[\mathrm{~J}]$. Jam Ceram Soc, 78(2): 297-304.

\section{Journal of Mines, Metals \& Fuels Please renew your subscription}

For details,contact : e-mail: bnjournals@gmail.com 